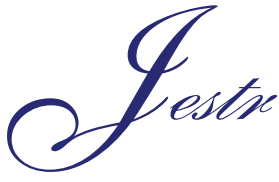

Journal of Engineering Science and Technology Review $3(1)$

Research Article
JOURNAL OF

Engineering Science and

Technology Review

\title{
R.ox.an.e: An Embedded System for Search and Rescue of Trapped in the Ruins of an Earthquake
}

\author{
D. Pogaridis, G. Karpathios, A. Pantelis and D. Karampatzakis
}

Kavala Institute of Technology, Department of Industrial Informatic, Kavala, 65404, Greece.

Received 8 March 2010; Accepted 30 September 2010

\begin{abstract}
R.ox.an.e is an embedded system for search and rescue of trapped victims in the ruins of an earthquake. A rescue team could use such kind of digital assistants in order to have quick and safe information about the disaster. The proposed system is a small size vehicle, with microcontroller based hardware and is wirelessly controlled. The operator has full control of the vehicle and is able to capture real time image of the accident place and various sensors measurements.
\end{abstract}

Keywords: Embedded Systems, Search and Rescue, wireless control, microcontroller, sensors, activators.

\section{Introduction}

An earthquake, beyond the destruction of the building infrastructure of the region where it takes place and the likely death of many citizens, it can trap many others in the ruins that it leaves behind. The search and rescue of the trapped is a very crucial and important matter.

This work presents the design, implementation and testing of a real time embedded system, called "R.ox.an.e", which aims at the localization of the surviving of trapped in the ruins after an earthquake [1]. The proposed system is a search and rescue robot, with wireless control and minimum electronic hardware capabilities. This kind of robots and the related scientific domain became hot after serious catastrophic situations all over the world $(9 / 11$, Katrina, Aquilla disasters) [2].

All the other systems use tiny operating systems or Windows, complicated algorithms and focus their interest to create autonomous robots which are compatible with the Robocup Rescue standards [3]. Because of low budget and our hardware background, our system is wireless controlled, with low level microcontroller software and a variety of sensors and activators. Our perspective is focused on low cost, small size, fast response and human-controlled robotic vehicles. Those robots could have quickly and safely assess of the overall situation and identify areas that have the lowest risk of danger to rescuers versus live victims.

The rest of the paper is organized as follows. The next section presents the system architecture and shows the main capabilities. Section 3 gives in more detail information about the hardware architecture and the most important hardware blocks. Section 4

*E-mail address: dpogaridis@teikav.edu.gr

ISSN: 1791-2377 (C) 2010 Kavala Institute of Technology. All rights reserved. describes the software architecture of the presented embedded system and fifth section presents project management procedures, testing results, and the final system figures. Finally, section 6 gives the overall concluding remarks.

\section{System Architecture}

The system is constituted of a Control Centre and a wireless controlled robotic Vehicle, as shown in Figure 1. The Control Centre provides the operator of the system with the ability to control the Vehicle from a distance and get the necessary audiovisual information, via a $\mathrm{LCD} 7^{\prime \prime}$ screen, a $\mathrm{CO}_{2}$ detector and a wireless bidirectional sound communication system, in order to localize and psychologically support the live victim until the rescue is over and done.

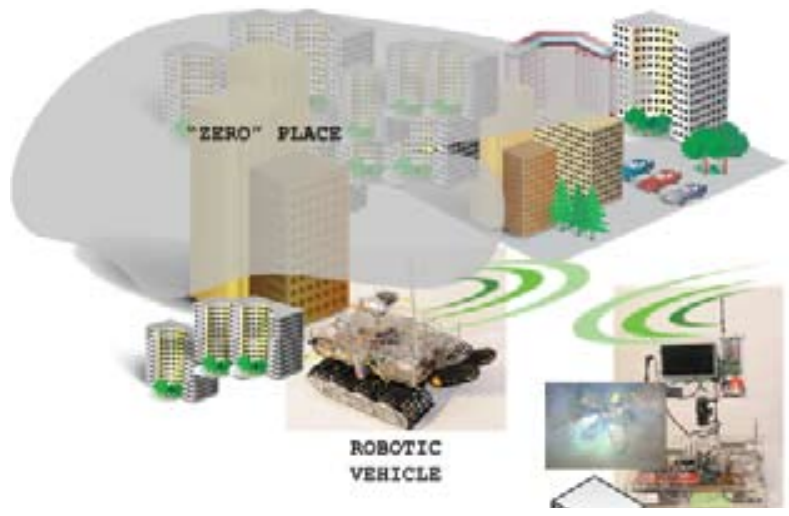

Figure 1. R.ox.an.e: System Architecture. 
It also allows the rescuer, via two LCD displays, to know, at any time, the functional state of the vehicle and the duration of the mission. The Vehicle is equipped with a camera, a microphone, a loudspeaker, a carbon dioxide detector, a smoke detector, a light detector and an inclinometer so that it can move through the ruins and collect information that concerns the localization of the victim. This information is transmitted to the Control Centre and delivered also to the internet.

Moreover on the Vehicle there is a fire tracing and extinguishing system, a sound recording system, a GSM transmitter that provides the position of the Vehicle via satellite. The Vehicle provides the possibility of self-test of its sensors and activators via internet and local network.

\section{Hardware architecture}

The system is constituted of two subsystems namely the Control Centre and the Vehicle. The Control Centre, shown in the block diagram of Figure 2, provides the operator with the necessary audiovisual information about the localization of the survivor and the benefit of psychological support until the rescue is done and over.

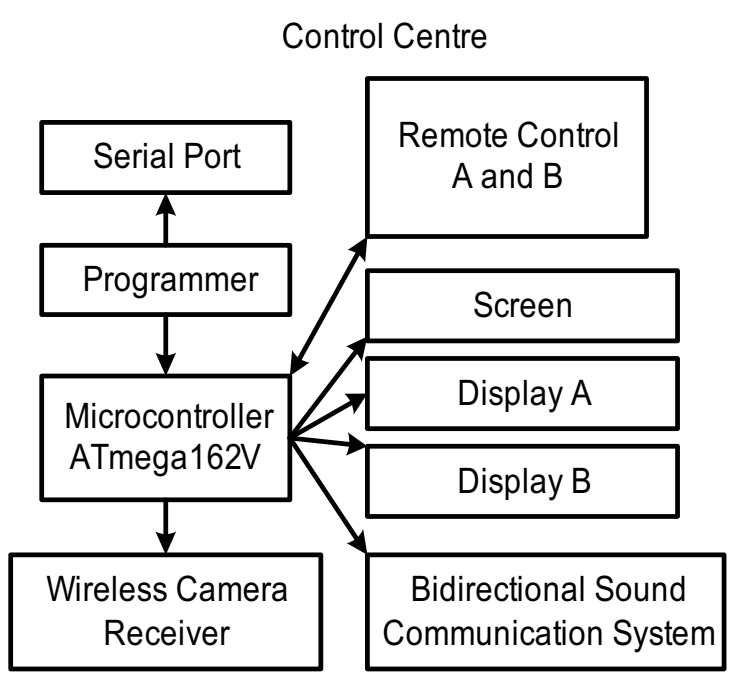

Figure 2. Hardware Architecture of the Control Centre.

To achieve that the Control Centre includes:

1. Two remote controls A and $\mathrm{B}$. Control $\mathrm{A}$ is used to drive the Vehicle and control B is used to control the camera on the Vehicle.

2. One LCD 7" screen which shows the picture captured by the camera.

3. One bidirectional sound communication system for acoustic communication with the trapped survivor.

4. One $(2 \times 16)$ LCD Display (A) and one alphanumeric LCD Display (B) that provides the system operator with the necessary information concerning the functionality of the Vehicle during the mission, so that it can be withdrawn when necessary.

5. Incorporated system programmer and battery charging system.

6. The Control Centre is controlled by an ATmega162V mi- crocontroller.

As soon as the Control Centre is powered up the microcontroller initializes it, by activating the receiver of the wireless camera, the wireless sound transmitter-receiver system, the screen and the remote controls A and B. All this procedure is shown on Display A. During initialization the system operator can check via remote control $\mathrm{B}$ the remote controls $\mathrm{A}$ and $\mathrm{B}$.

The upgrading of the software of the Control Centre and the Vehicle can be done through an incorporated splitter and a serial system I/O programmer (Serial Input/Output).

The Control Centre is powered by rechargeable lithium batteries, which are charged by an incorporated charging circuit. The value of the battery voltage and the duration of the mission at any time are shown on Display B.

The motion of the Vehicle and the camera are controlled from the Control Centre through the remote controls A and B and a six channel transmitter-receiver system [6], which transmits at $35 \mathrm{MHz}$ and is shown in the block diagram of Figure 3.

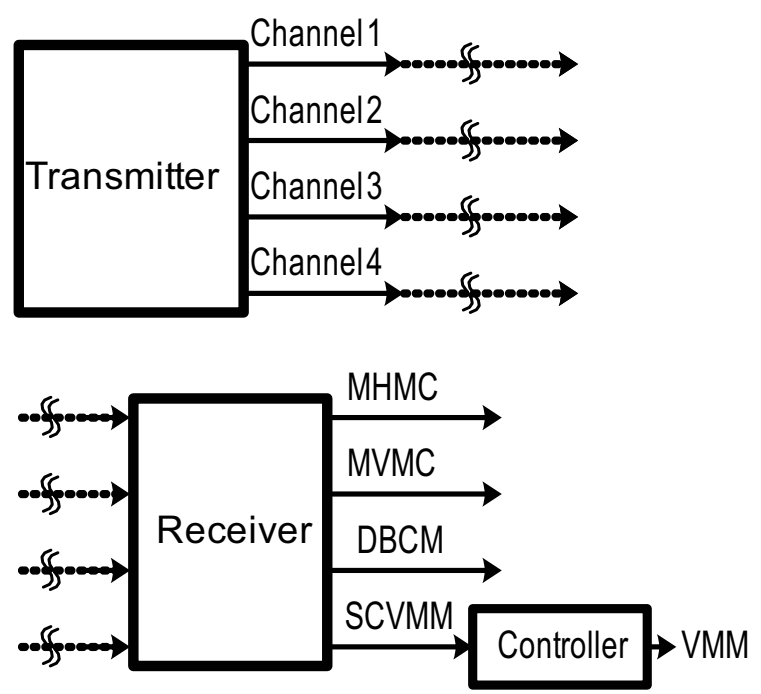

Figure 3. Block diagram of the remote control system of the camera and the Vehicle.

The transmitter is activated by the microcontroller via a relay, gets information about the control of the motion of the Vehicle and the camera from the remote control A and B respectively and transmits it to the receiver. The receiver handles this information and performs the corresponding operations. This information is shown in real time, through the microcontroller of the Control Centre, on Display A. The Vehicle, shown in Figure 4, is equipped with:

1. A night vision camera and the Transmitter.

2. A bidirectional sound communication system.

3. Light, smoke and carbon dioxide detectors.

4. An inclinometer.

5. A sound recording system.

6. A GSM transmitter.

7. An incorporated battery charging system.

8. The Vehicle is controlled by an ATmega162V microcontroller. 


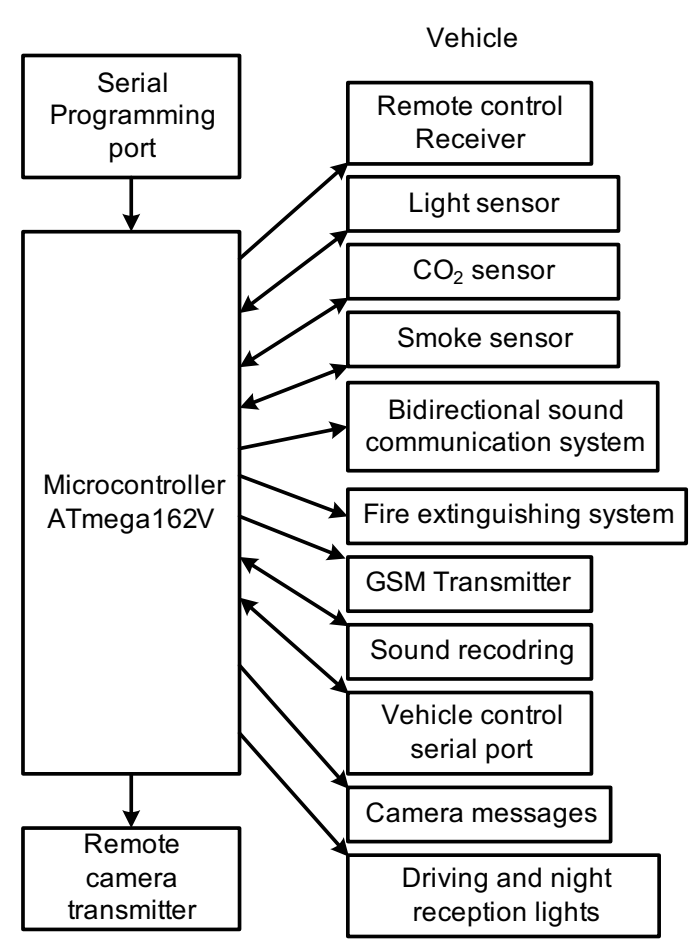

Figure 4. Hardware Architecture of the Vehicle.

The microcontroller, as soon as the Vehicle is powered up, enables the receiver of the remote control system that concerns the motion of the Vehicle, the light detector, the carbon dioxide detector, the smoke detector, the bidirectional audio communication system, the transmitter of the wireless camera, the sound recording system and the GSM transmitter. During this process the system operator is able to check the functionality of all the detectors via a push button. At the same time, via a switch, enables or not the recording of the sounds during the mission. After this procedure the carbon dioxide sensor [5] records the value of carbon dioxide contained in open air. This measurement is used as a reference value during the mission.

The Vehicle moves using a pair of miniature tank tracks with large aperture allowing it to move with large lateral gradients without being overturned. The tank tracks are powered by a DC motor with speed controller [6], as shown in Figure 3. On the tank tracks is adapted a differential axle, which in combination with two disc brakes that have been adapted on it, enables rotation of the Vehicle up to the $360^{\circ}$. When the Vehicle moves uphill with slopes greater than the $45^{\circ}$ then automatically, a mercury inclinometer mounted on the Vehicle, detects the slope and starts a second DC motor, which powers a second smaller rear pair of tracks in order to prevent Vehicle overthrow.

On the Vehicle is positioned a night vision camera which is controlled by two servomotors to allow $180^{\circ}$ horizontal and $90^{\circ}$ vertical rotation. The image of the camera, the night vision led indicators, smoke, and carbon dioxide detectors, the low-level voltage of the battery of the Vehicle are transferred wirelessly to the system operator. They are shown on the screen of the Control Centre and help the operator to direct the Vehicle to the survivor. The same information is streamed simultaneously to the internet and to the crisis management centre.
The embedded in the camera microphone and a speaker on the Vehicle allow bidirectional sound communication and this in turn allows the system operator to locate the survivor through his voice and to provide the necessary psychological support until the rescue. A GSM transmitter enables positioning of the vehicle through satellite.

Finally, on the Vehicle there is an autonomous smoke detection [7] and a small-scale fire extinguishing system controlled by an ATtiny 13 microcontroller. This microcontroller in cooperation with the main microcontroller of the Vehicle displays on the screen of the Control Centre the indication of smoke detection.

\section{Software architecture}

The low level embedded software was written in C language, using bitmaps and bitwise allowing the management of characters in bit level. All the philosophy of the program is based on interrupts. So, the microcontroller responds in real time to the interrupt requests of the detectors the other parts of the system.

The software of the Control Centre, shown in the flow diagram of Figure 5, starts with activating its peripherals while at same time shows the corresponding messages on Display A. After that the program gets into an endless loop waiting for interrupt requests, while at the same time displays some messages on Display A.

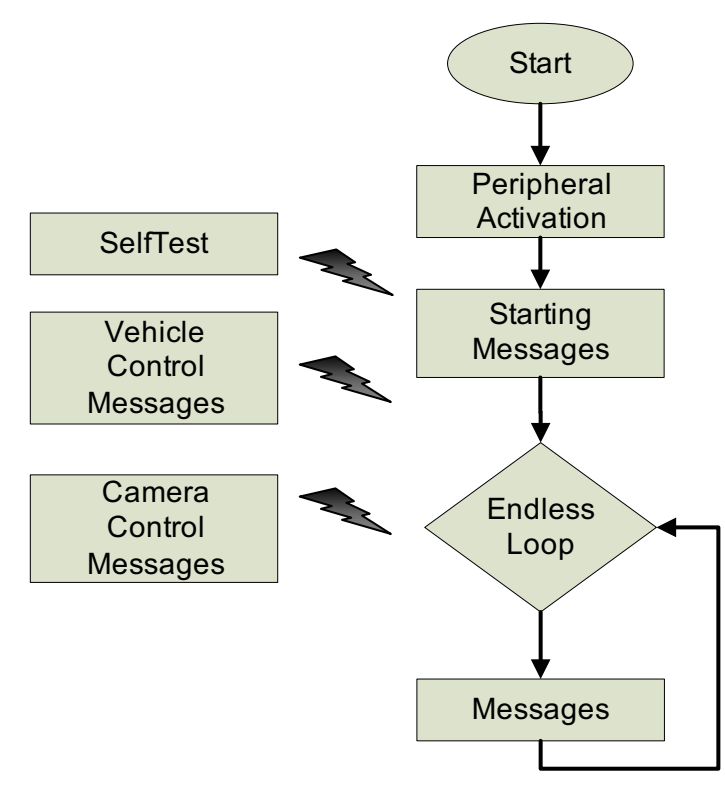

Figure 5. Flow diagram of Control Centre software.

The interrupt requests may occur from the following sources:

1. Interrupt request for self-test of the Control Centre that occurs from the remote control of the camera during the activation of the peripherals.

2. Interrupt request for displaying the messages concerning the navigation of the Vehicle. This request comes from the remote control of the Vehicle.

3. Interrupt request for displaying the messages concerning the motion of the camera. This request comes from the remote control of the camera. 
The software program of the Vehicle, shown in the flow diagram of Figure 6, starts with the activation of its peripherals and then enters in an endless loop waiting for interrupts. Inside this loop checks the state of the switch, that enables the sounds recording system when low, and if found high, in the case the system has been enabled before the beginning of the mission, it will store the sound information together with the date and time into a file.

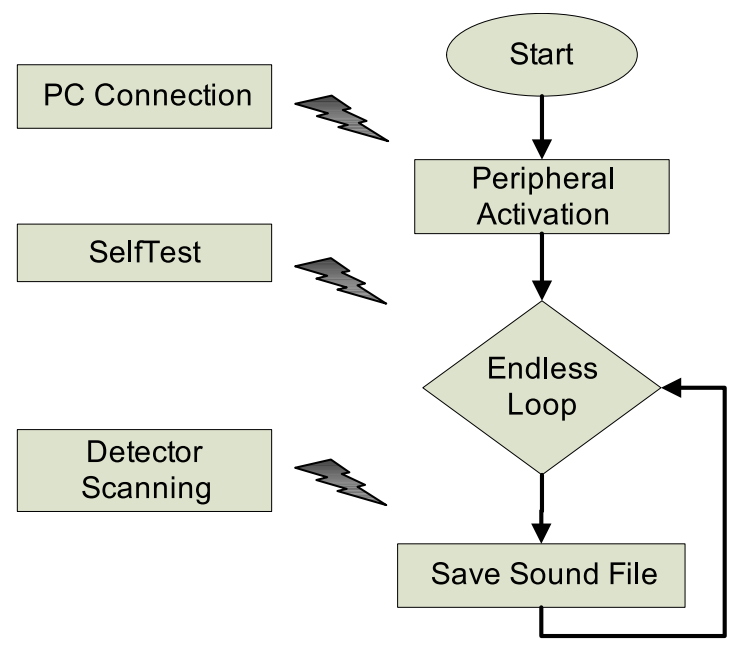

Figure 6. Flow diagram of Vehicle software.

The interrupt requests may occur from the following sources:

1. Interrupt request for immobilizing the Vehicle when it is connected to a computer for the withdrawal of the audio documents which have been recorded during the mission.

2. Interrupt request for self-test of the Vehicle that can come either from a push button on the Vehicle or from the internet through the serial Vehicle control port.

3. Interrupt request from the light, carbon dioxide and smoke detectors.

The software programs, as mentioned, can be upgraded using the serial programmer.

\section{System testing and project management}

During the project deployment the system requirements were categorized according to the FURPS (Functionality Usability Reliability Performance Supportability or FURPS) [8] model. The Gantt chart [9] was also used in representing the phases and activities of the project.

Full open and closed box tests took place [10, 11]. During the open box tests the response of all the parts were tested for good operation. During the closed box tests all the individual functions of the program, that were recorded as nodes, were checked and following the way each variables makes its ways in the source code was determined.

The paths and loops that were created, as well as the behaviour of each variable inside these paths and loops, were recorded. Because the present system is a real time application the closed box tests were limited.
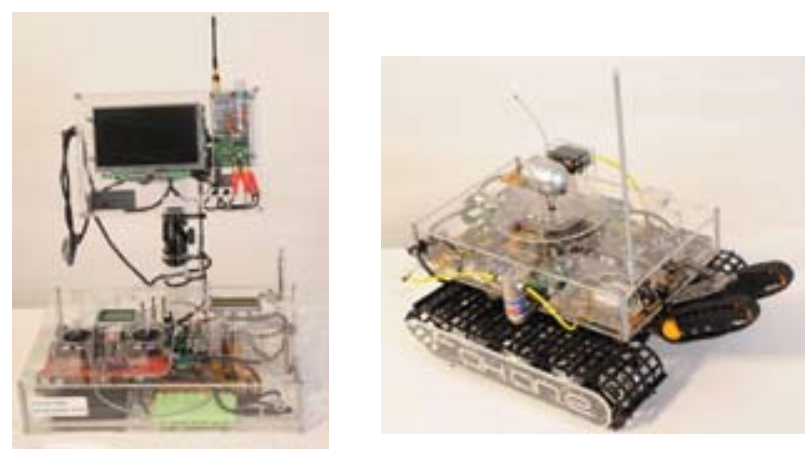

Figure 7. The Control Centre and the Robotic Vehicle.

The voltage supply and incorporated battery charging system also tested. The system uses two LiPo batteries for maximum mission duration. Real world tests shows that the main power consumption is coming from the DC motors used for the Vehicle motion. Based on these results we plan to design a more lightweight version of the Vehicle's mechanical parts in order to use less power hungry motors.

The final implementation of the system "R.ox.an.e" is shown in Figure 7, and consists of the Control Centre and the Robotic Vehicle.

\section{Conclusions}

A complete prototype of a search and rescue system presented to this work. The system is based on an embedded architecture, equipped with various sensors and a camera. R.ox.an.e is especially designed for situations after earthquakes and physical disasters in order to provide important information for the accident place. Based on this prototype our team will work in order to improve the main parts of the system and to enhance the operational services.

\section{Acronyms used in the work.}

Vertical Rotation Camera Motor or VRCM

Horizontal Rotation Camera Motor or HRCM

Vehicle Motion Motor or VMM

Speed Control Vehicle Motion Motor or SCVMM

Disc Brake Control Motor or DBCM 


\section{References}

1. G. Karpathios, A. Pantelis, «An embedded system for the Localisation of Trapped in the Ruins of an Earthquake», Final Year Project, Department of Industrial Informatics, Kavala Institute of Technology, 2009.

2. SRMED, International Workshop on Synthetic Simulation and Robotics to Mitigate Earthquake Disaster.

3. Robocup Rescue, http://www.robocup2009.org.

4. ART-TECH R/C Hobby Co., Ltd, ShenZhen.

5. CDM4161 - Precalibrated module for carbon dioxide, Figaro Engineeing Inc. 1-5-11 Senbanishi, Mino, Osaka 562 Japan, http://www.figaro.co.jp.

6. Special DC motors, http://www.lrp.cc.

7. Ionized Smoke Sensor, http://img.diytrade.com.

8. K. Khosravi, Y. Gueheneuc, «A Quality Model for Design Patterns», Technical Report 1249, University of Montreal, 2004.

9. H. L. Gantt, Work, Wages and Profit, published by The Engineering Magazine, New York, 1910.
10. H. D. Mills, R. C. Linger. A. R. Herner, «Box structured information systems», IBM Systems Journal, Volume 26, issue 4, 1987.

11. H. D. Mills, R. C. Linger, A. R. Hevner, Mathematical aspects of box structures, Proceedings of the Twenty-First Annual Hawaii Inter-national Conference on Software Track, January 1988, IEEE Computer Society Press.

12. D. Pogaridis, «Embedded Systems. The AVR microcontrollers», V. Giourdas Publishing, forthcoming, 2009.

13. D. Pogaridis, «Microprocessor Design Systems», V. Giourdas Publishing, 2006.

14. D. Pogaridis, «Digital Design with VHDL Language», V.Giourdas Publishing, 2007.

15. E. Nikolaropoulos, Course Notes «Computer based systems», Department of Industrial Informatics, Kavala Institute of Technology. 